Original Research Paper

\title{
Extended-Spectrum $\beta$-Lactamases Producing Escherichia coli Strains Monitored Over 4 Years in The University Hospital in Košice, Slovakia
}

\author{
${ }^{1}$ Viera Lovayová, ${ }^{2}$ Lucia Vargová, ${ }^{2}$ Mária Nagyová, \\ ${ }^{3}$ Eva Dudriková, ${ }^{1}$ Erik Dorko and ${ }^{2}$ Leonard Siegfried \\ ${ }^{I}$ Department of Public Health and Hygiene, Faculty of Medicine, University of P.J. Safarik in Kosice, Slovakia \\ ${ }^{2}$ Department of Medical and Clinical Microbiology, Faculty of Medicine, University of P.J. Safarik in Kosice, Slovakia \\ ${ }^{3}$ Department of Food, Hygiene and Technology, University of Veterinary Medicine and Pharmacy in Kosice, Slovakia
}

Article history

Received: 18-01-2016

Revised: 14-06-2016

Accepted: 15-06-2016

Corresponding Author:

Viera Lovayová

Department of Public Health

and Hygiene, Faculty of

Medicine, University of P.J.

Safarik in Košice, Trieda SNP

1, 04011 Košice, Slovakia

Phone: +421915932031

Email: viera.lovayova@upjs.sk

\begin{abstract}
The beta-lactamases with extended spectrum of activity (ESBL) are medically one of the most important group of enzymes. The presented study provides identification and determination of the spectrum of resistance against different and clinically used antimicrobial drugs in the clinical isolates of Escherichia coli. These isolates had their origin in different departments of the University Hospital L. Pasteur in Košice. The second goal was the detection of beta-lactamase production with extendedspectrum effect (ESBL) and testing of AmpC-type cephalosporinases by several phenotypic tests in clinical isolates. We used both the microdilution method and the method with an active agent, respectively. Samples were positively tested for ESBL with the use of the CLSI disk diffusion method. PCRs were performed with a series of primers designed for the detection of Ambler class A, B and C beta-lactamase genes. About 307 strains of E. coli were investigated. The growth of $E$. coli resistance to selected antibiotics was present in $83.25 \%$ of clinical isolates. There were identified 85 positive isolates in the studied group and the prevalence of the ESBL positive strains of $E$. coli reached $27.78 \%$. An E. coli strain was isolated with mutations in the promoter region of the AmpC chromosomal gene that is associated with overproduction of the relevant enzyme. We describe a complex ESBL epidemiology. The study revealed a high rate of ESBL-producing E. coli isolates. The $b l a_{\mathrm{TEM}}$ and $b l a_{\mathrm{SHV}}$ enzymes dominated in ESBL-positive E. coli isolates in the University Hospital L. Pasteur in Košice.
\end{abstract}

Keywords: E. coli, Antimicrobial Resistance, ESBL, Clinical Isolates, Prevalence

\section{Introduction}

One of the most serious problems in medicine today is bacterial infection, the role of which has increased dramatically in recent years. The prevalence of extended-spectrum beta-lactamase (ESBL-producing Enterobacteriaceae) is increasing globally and community-onset infections with ESBL-producing Escherichia coli are a major clinical concern in many countries (Laupland et al., 2008).

Many genera of gram-negative bacteria possess a naturally occurring, chromosomally mediated $\beta$ lactamase. These enzymes are thought to have evolved from penicillin-binding Proteins, with which they show some sequence homology. This development is likely due to the selective pressure exerted by $\beta$-lactamproducing soil organisms found in the environment (Ghuysen, 1991). The most common cause of resistance to expanded-spectrum cephalosporins in E. coli is the production of Extended-Spectrum $\beta$-Lactamases (ESBLs) (Paterson, 2006). In the past decade, CTX-Mtype ESBLs have replaced TEM- and SHV-type ESBLs in Europe, Canada, Asia (Apisarnthanarak et al., 2007), South America (Bonnet, 2004) and North America (Moland et al., 2003) as the most common ESBL type in this species.

In addition, the expansion of the active site that allows the increased activity against expanded-spectrum 
cephalosporins may also result in the increased susceptibility of ESBLs to $\beta$-lactamase inhibitors (Jacoby and Medeiros, 1991). ESBLs are not active against cephamycins and most strains expressing ESBLs are susceptible to cefoxitin and cefotetan. However, it has been reported that ESBL-producing strains can become resistant to cephamycins due to the loss of an outer membrane porin protein (Martine'z-Martine'z et al., 1996).

The aim of the paper is to identify and determine the spectrum of resistance against a variety of clinically used antimicrobial pharmaceuticals for clinical isolates of the genus $E$. coli from different departments of the University L Pasteur Hospital in Košice, Slovakia and the detection of $\beta$-lactamase production with extended range (ESBL) and clinical isolates tested in multiple phenotype Cephalosporinases types of ESBLs and genotype tests.

\section{Materials and Methods}

In the years 2009-2012 strains of Escherichia coli were isolated from clinical material (urine, swab from the throat, swab from the wound, swab from decubitus, a swab of the cervix, etc.), which were collected from at various departments of the University L. Pasteur Hospital in Košice.

\section{Bacterial Isolates}

A total of 307 samples of the strains were examined. MALDI-TOF MS analysis was performed on a Microflex MALDI Biotyper (Bruker Daltonik) according to a standard sample preparation protocol of Bruker Daltonik (Freiwald and Sauer, 2009). MALDI-TOF mass spectra were subjected to numerical analysis (BioTyper 3.1 software, Bruker Daltonik).

ESBL production was detected in $85(27.7 \%)$ E. coli isolates recovered from 307 samples. Samples were collected from the following departments: Department of Internal Medicine 17 (5.5\%), Department of Surgery 90 (29.6\%), Department of Anaesthesiology and Intensive Medicine 39 (12.7\%), Department of Haematology and Oncohaematology $22(7.1 \%)$, Department of Urology 16 $(5.2 \%)$ and other $123(39.9 \%)$.

E. coli strains were confirmed by standard biochemical methods ENTERO test 16 and ENTERORapid 24 (Lachema, Czech Republic).

\section{Susceptibility Testing}

The isolates were tested for antimicrobial susceptibility using the disk diffusion method according to the Clinical and Laboratory Standards Institute (CLSI) guidelines (CLSI, 2009a).

In the case of all isolates with a Minimum Inhibitory Concentration (MIC) in at least one test of the 3rdgeneration Cephalosporins (Cephotaxime, Cephtasidime or Cephoperasone) of more than $1 \mathrm{mg} \mathrm{L}^{-1}$, a modified Double Disk Synergy Test (DDST) was carried out combined with the method for the ESBL production CLSI determination. Apart from that, the detection of carbapenemases by the modified Hodge test was used.

The quantitative susceptibility Minimum Inhibitory Concentration (MIC) of Enterobacteriaceae isolates was determined antibiotic diagnostic The MIDITECH system automated colorimetric test for antimicrobial susceptibility testing. The principle of identification is consistent with the standard microdilution method used to identify antibiotic susceptibility (CLSI, 2006). The test meets the quality requirements of the CLSI standard (Clinical and Laboratory Standards Institute) (CLSI, 2009b; 2011).

\section{PCR Amplification and Sequencing}

The detection of Extended Spectrum Beta-Lactamase (ESBL) genes was carried out by PCR with specific primers to detect bla $_{C T X-M}$, bla $_{S H V}$, bla $_{T E M}, A m p C_{C I T}$ genes (Howard et al., 2002). The following primers were used in these reactions: for bla $\mathrm{T}_{\mathrm{TEM}}$, primers forward: TEM-A (5' CCCCGAAGAACGTTTTC '3) and reverse: TEM-B ( 5 ' ATCAGCAATAAACCAGC ' 3 ), fragment length is

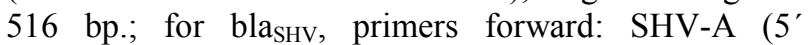
TCAGCGAAAAACACCTTG '3) and reverse: SHV-B (5' TCCCGCAGATAAATCACCA '3), fragment length is $475 \mathrm{bp}$; for bla $\mathrm{ClX}_{\mathrm{CTX}}$, primers forward: CTX-MU1 (5'TCCGAGTTGACTGCCGGGTTG 3') and reverse: CTX-MU2 (5'TTACAAACCGTYGGTGAC3'), fragment length is $543 \mathrm{bp}$; and for Ampc CIT. primers forward: MF (5' TGGCCAGAACTGACAGGCAAA '3) and reverse: MR (5' TTTCTCCTGAACGTGGCTGGC ' 3 ), fragment length is $462 \mathrm{bp}$.

E. coli strain (CNCTC-7374) was used as the control strain in the detection of these genes. Genes coding ESBL enzymes (CTX-M, SHV-type and TEM-type) were studied by PCR and sequencing in all AMC-resistant $E$. coli isolates with phenotypes consistent with ESBL production on the basis of their resistance to the extendedspectrum cephalosporins whose activity was recovered in the presence of clavulanate (Oteo et al., 2006).

\section{Sequencing of TEM, SHV and CTX-M Genes}

The PCR bla $a_{S H V}, b l a_{T E M}$ and $b l a_{C T X-M}$ products were purified with the PCR SureClean Plus (Applied Biosystems) and sequenced with a Genetic Analyzer 3600 (Life technologies). The nucleotide sequences, deduced amino acid sequences and phylogenetic relationships were analyzed by using the software package (SeqScape v2.7 and MicroSeq v2.2).

\section{Isolation and Separation of Plasmid DNA}

Plasmid DNA was extracted from both donor and transconjugants. A small-scale alkaline lysis method was used as described by (Sambrook et al., 1982). Extracted 
plasmids were electrophoresed for $2 \mathrm{~h}$ in a horizontal $0.8 \%$ agarose gel with pH 8.0 TBE buffers (Plaziniski et al., 1985). The gels were stained with ethidium bromide 0.5 $\mu \mathrm{g} \mathrm{mL}{ }^{-1}$ for $20 \mathrm{~min}$. and bands were visualized by UV transilluminator. Lambda DNA digested with Hind 111 and EcoRI was used as the DNA standard marker.

\section{Statistical Evaluation}

For statistical comparison of the results, statistical methods of processing and evaluation of the results were used to compare data processed into the tables and graph (MS Excel 2010, IBM SPSS statistics 19).

\section{Results and Discussion}

Strains isolated from clinical material in the University L Pasteur Hospital in Kosice were compared in terms of susceptibility tests, results of phenotypic tests and tests for detection of genes coding ESBL. In four years 307 isolates of one of the E. coli species had been isolated from precisely the same number of patients with invasive infections. The largest part of the collected material came from urine and wound swabs. $16.94 \%$ of $E$. coli strains were collected from the 18-40 age group, $28.76 \%$ of the strains came from the $41-60$ age group and $54.49 \%$ of the $E$. coli positive samples came from patients of 61 years of age and older.

The median age of patients infected with an ESBLproducing isolate was 59 years (range 18-89 years) and a higher proportion of female patients (51.8\%) was infected with ESBL-producing E. coli (Table 1).

The nosocomial origin of the majority of monitored bacteria discussed in this study is also supported by the age spectrum of the patients. The incidence of infection was recorded among adults $(83.25 \%)$ of 41 years of age and older. E. coli resistance to the selected antibiotic also increased with age. The difference is evident even between genders where there is higher antibiotic resistance among women than men. Tendencies in resistance to selected antimicrobials in the monitored period (2009-2012) can be seen in Fig. 1.

The results shown in the Fig. 1 indicate that there is no clear tendency that can be traced showing antibiotic resistance deterioration of the $E$. coli strain within the variety of sampling material used. Differences found in resistance of E. coli strains to the antibiotics between the years are more random in nature and are most likely related to specific mass occurrences of certain E. coli strains with a greater degree of resistance in the particular years and seasons. It can be assumed that the cause of these differences is the use of antibiotics in various clinical circumstances.

Resistance to ampicillin was $59.23 \%$ on average each monitored year. Gradual growth of E. coli strain resistance to ampicillin was found in $2011(63.29 \%)$ followed by a slight decrease to $57.25 \%$ in 2012 .
Resistance of $E$. coli strains to Cephalosporins oscillates in the long term and was $39.82 \%$ in $2009,24.29 \%$ in 2010 and $23.73 \%$ in 2011. In 2012 the percentage dropped to $21.28 \%$ while resistance to QuinoloneCiprophloxacin decreased fast and consistently. In 2009 there were $51,85 \%$ cases of $E$. coli strains resistant to ciprofloxacin recorded, while in 2012 it was $34.35 \%$.

Resistance of the E. coli strain to Gentamycin compared to the resistance to Aztreonam in the course of a four-year monitored period was four times lower. Fluctuations were recorded in both cases. On the other hand, the $E$ coli strain was sensitive to carbapenems (meropenem) in all cases.

In determining the production of ESBL by means of the dual-disk diffusive test there were 85 positive isolates identified in the tested set. Based on the results, it is clear that the prevalence of the ESBL positive $E$. coli strains reached $27.78 \%$.

When comparing the occurrence of ESBL E. coli positive strains across the genders, the results showed 1.29 times $(\mathrm{OR}=1.29)$ higher risk of ESBL positive $E$. coli strains occurrence in men compared to women. The occurrence of ESBL positive E. coli strains based on a statistical gender evaluation was insignificant at $95 \%$ confidence level, (the statistical $\mathrm{p}<0.3682$ ).

Tests to detect ESBL might be misleadingly positive if the strain produces the narrow spectre $\beta$-lactamase (TEM-1,2) simultaneously with the AmpC. In this case a test on the AmpC with Tris-EDTA disk (Munier et al., 2010) is done.

According to Bradford (2001) there is a common occurrence of resistance against most of the $\beta$-lactamase antibiotics related to the production of various types of $\beta$-lactamase antibiotics with extended effect spectrum, hydrolysing Penicillins, Cephalosporins of the first, second and third generation as well as monobactams. Out of a total number of 85 isolates with confirmed ESBL production $60(70.59 \%) E$. coli clinical isolates were evaluated as multi-drug resistant.

ESBL-producers' sensitivity to Cephalosporins was relatively low (24.71\%). 93.33\% of aminoglycoside gentamicin also showed high efficiency. For all the ESBL producers the sensitivity to Carbapenems (meropenems) has been preserved (100\%). These substances belong to the last choice antibiotics and therefore the incidence of antibiotic-resistant clinical isolates against them remains low (Pages et al., 2005). In the therapy of infections caused by ESBL producers these substances are among the most effective medicines (Nordmann and Poirel, 2002).

The most precise method used to determine the cephalosporinases production of the AmpC type was the double disk diffusion test which detected three (1.7\%) positive clinical isolates. Slightly increased results were recorded also by the authors Sidjabat et al. (2009) who identified 2.8\% of the AmpC-producing isolates. 


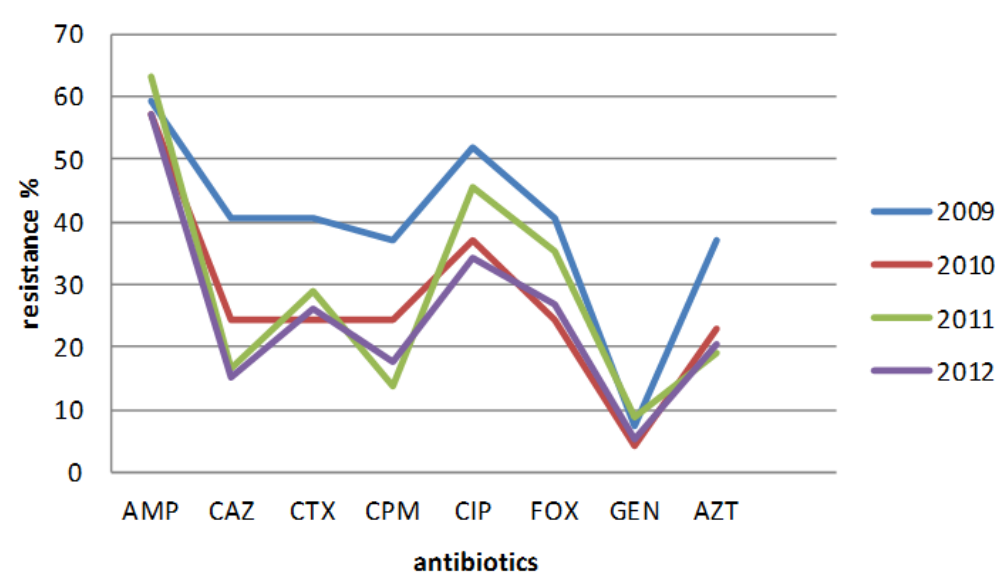

Fig. 1. Trends in antibiotic resistance of different antibiotics in E. coli strains isolated during the period 2009-2012

Table 1. The incidence of ESBL-positive strains of E. coli in the period 2009-2012

\begin{tabular}{|c|c|c|c|}
\hline \multirow[b]{2}{*}{ Gender } & \multicolumn{3}{|l|}{ ESBL } \\
\hline & Positive (n) & Negative (n) & All (n) \\
\hline$\overline{\text { Men }}$ & 41 & 93 & 134 \\
\hline Women & 44 & 129 & 173 \\
\hline Together & 85 & 222 & 307 \\
\hline
\end{tabular}

Only one sample produced ESBL and AmpC simultaneously. According to Coudron et al. (2000) AmpC type cephalosporinases make bacteria resistant against a wide range of $\beta$-lactamase antibiotics including Cefoxitin and other broad spectrum antibiotics including cephalosporins, Aztreonam and combinations of $\beta$ lactams with $\beta$-lactamase inhibitors.

By applying new EUCAST/CLSI limits when interpreting the results of examination carbapenems sensitivity, all producers of clinically significant carbapenemases should be detected. Over the course of 4 years, the number of positive E. coli samples sensitive to Carbapenem-Imipenem was, on average, $18.85 \%$ of the total 307 samples. The highest number of positive $E$. coli samples sensitive to Carbapenem-Imipenem was recorded in $2011(25.31 \%)$.

Within the monitored period, the number of $E$. coli samples positive on the Carbapenem-Ertapenem was, on average, was $10.65 \%$, lower than in case of the antibiotic Imipenem. In 2011 the highest sensitivity to antibiotic Ertapenem as compared carbapenems (18.99\%) was recorded.

Ertapenem, the only representative of Carbapenems yet registered in Slovakia, is ineffective against Pseudomonas aeruginosa and the Enterococci (Edwards et al., 2005).

The PCR method has identified the genes responsible for the antibiotic resistance to $\beta$-lactam antibiotics. During the monitored period we have proven at least 1 resistance gene out of 307 resistant E. coli strains tested in 85 positive isolates. These positive isolates were subjected to the PCR method to detect the presence of bla $a_{\mathrm{TEM}}, b l a_{\mathrm{SHV}}, b l a_{\mathrm{CTX}-\mathrm{M}}$ and $A m p C_{\mathrm{CIT}}$ genes coding $\beta$ lactamase or a wide Spectral $\beta$-Lactamase (ESBL).

Of the $\beta$-lactamase coded by bla genes such as TEM, SHV and CTX genes, 120 (39.1\%) bla $a_{\mathrm{TEM}}$ E. coli genes and $42(13.7 \%) b l a_{\mathrm{SHV}}$ genes were detected out of $307 \mathrm{E}$ coli clinical isolates. About 24 (7.8\%) bla $a_{\text {СтХ-м genes }}$ were detected out of the total number of 307 clinical isolates. From a group of $\beta$-lactamases, AmpC enzymes hydrolysing $\beta$-lactam antibiotics 31 (10.1\%) of $A m p C_{\mathrm{CIT}}$ genes have been confirmed among clinical isolates of $E$. coli. Belgian authors Bogaerts et al. (2009), as documented in their paper, found that out of a total number of 83 AmpC positive clinical isolates of $E$. coli an increased expression of $\mathrm{AmpC}$ gene encoded on chromosome was observed in 72 cases. For the remaining $11 \mathrm{E}$. coli isolates, in which the enzyme overproduction had not been confirmed, the presence of plasmid-encoded AmpC $\beta$-lactamases was detected.

In accord with the trend of $\beta$-lactamase ESBL gene presence, an increase in $b l a_{\mathrm{TEM}}$ genes was recorded in the 4-year monitored period. In 20098 strains, 29.63\%, with the $b l a_{\mathrm{TEM}}$ genes were recorded. An increase to 29 strains, $41.43 \%$, was detected in 2010 and $40 \mathrm{E}$. coli strains, $50.63 \%$, in 2011. In 2012 the number of strains with the $b l a_{\text {TEM }}$ genes was $43,32.82 \%$ of the total number of 131 collected $E$. coli strains.

The tendency to increase with sporadic presence has been recorded in $E$. coli strain $b l a_{\mathrm{SHV}}$ genes. The increase in $A m p C_{\mathrm{CIT}}$ genes in clinical isolates was moderate in the 4-year monitored period, but in 2010 there was a great increase in comparison to the other years with 16 $(22.86 \%)$ positive strains containing the $A m p C_{\mathrm{CIT}}$ gene. In 2012, no incidence was reported concerning $A m p C_{\mathrm{CIT}}$ genes in E. coli positive strains. AmpC gene expression is constitutive in $E$. coli under normal specifications; the $\beta$-lactamase is produced in very small quantities. 
In the course of the analysis of genes encoding $\beta$ lactamase production in E. coli strains isolated from individual sampling materials, it was found that $b l a_{\mathrm{TEM}}$ gene was the most prominent in urine samples of 41 patients $(41.84 \%)$ and wound swabs of 29 patients (36.25\%), of which the most frequently resistant $E$. coli strains were isolated. The slightly higher representation of the $b l a_{\text {SHV }}$ gene was found in biological samples from urine in 19 patients $(19.39 \%)$ as well as in wound swabs of 10 patients $(12.8 \%)$. The overview of genes encoding $\beta$-lactamase production in clinical samples coming from different sampling material concerning $E$. coli is given in Table 2 .

The highest prevalence of $b l a_{\mathrm{TEM}}, \quad b l a_{\mathrm{SHV}}$ and $A m p C_{\mathrm{CIT}}$ genes was present in the samples taken from patients with digestive system diseases or urethral venereal diseases. The most resistant isolated E. coli strains came from Surgical Clinic patients and the patients in the Department of Internal Medicine, where most of the patients require antibiotic treatment. Therefore there is an increased risk of the occurrence and development of antibiotic resistance.

The risk concerning the patients lying at the surgical ward is higher in the age group of 61 years and over at the value of 1.20 and a $95 \%$ level of confidence.

The statistical evaluation of the OR for the presence of ESBL E. coli positive strains in the group of men suffering from gastrointestinal disorders compared to women was $0.73(95 \% \mathrm{CI})$ and the results based on the Fisher's test was not significant. When assessing each Department, patients at Surgery Department have 0.44 times higher risk of ESBL positive E. coli strains than the patients at Internal Department. Furthermore the results showed an increase of the ESBL positive strains depending on the gender at Surgery Department, where there is a $63 \%(0.48 ; 95 \% \mathrm{CI}=\mathrm{OR})$ chance of men contracting ESBL positive strains and a $55 \%(\mathrm{OR} 95 \% \mathrm{CI}=0.39)$ chance for women staying in the Surgery Department.

The level of risk in relation to the sampling material has an increasing trend for women, which is two times higher than the risk of ESBL positive E. coli strains in the urine $(\mathrm{OR}=2.489 ; 95 \% \mathrm{CI})$. Statistical analysis confirmed the risk of ESBL positive strains occurrence in the urine in females compared with the previous sample material, which demonstrated a level of significance of $95 \%(p<0.05)$. From the anatomical point of view, women's urine may be more contaminated due to the surrounding flora, either rectal or vaginal.

Results showed differences not only between genders, but also between different age groups. The level of risk in relation to age and diagnosis shows the tendency of increased risk of contraction with regards to age-it is more prevalent in the age group of 18-40 yearold patients; the proportion of the risk of ESBL E. coli positive strains is double in comparison to other age groups $(\mathrm{OR}=2.18 ; 95 \% \mathrm{CI})$. Comparison of the risk tendency in relation to age in the course of the monitored years is shown in Fig. 2.

Control of the spread of infectious diseases is probably one of the main tasks of contemporary medicine.

It has been shown that there is an increase in prevalence of drug resistance among $E$. coli isolates in our region and majority of the antibiotic resistances were due to the acquisition of plasmid-carrying antibioticresistance genes. Conjugal transfer of plasmids has greatly contributed to the rapid spread of antibiotic resistance among E. coli isolates. Restriction of use of antibiotics may play a role in decreasing the emergence of resistant bacterial strains.

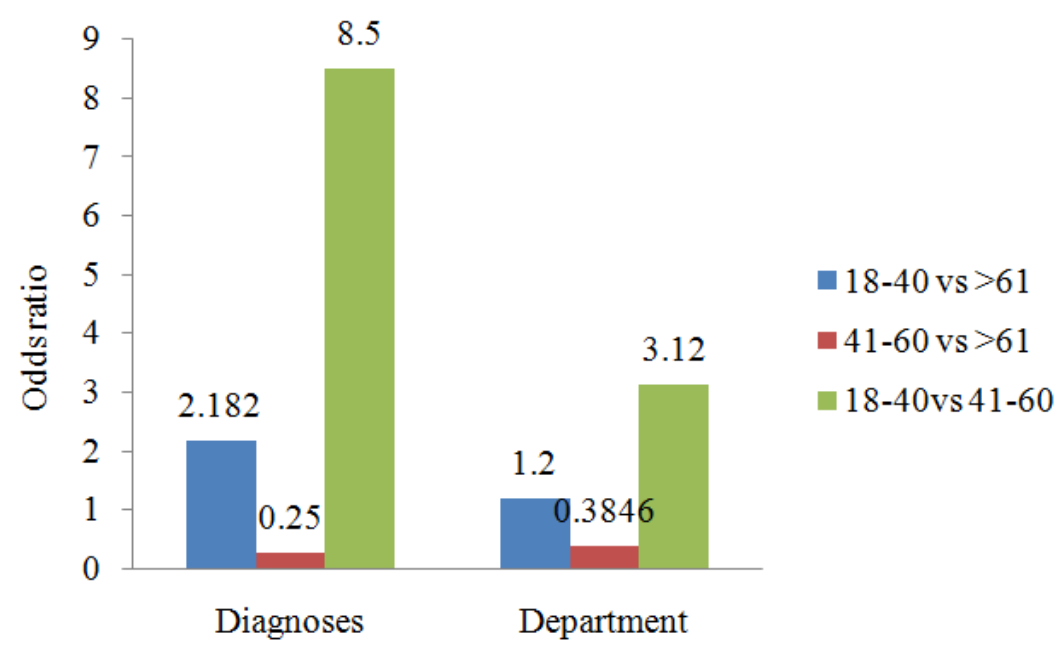

Fig. 2. Examination of the trend of risk in relation to age in the monitored years 
Table 2. Incidence of genes encoding the formation of $\beta$-lactamases from various clinical specimens for E. coli $\mathrm{ESBL}$, AmpC strains in the period 2009-2012

\begin{tabular}{|c|c|c|c|c|}
\hline \multirow[b]{2}{*}{ Clinical material $(n=307)$} & \multicolumn{4}{|c|}{ Detected resistance genes } \\
\hline & $b l a_{\mathrm{TEM}}$ & $b l a_{\mathrm{SHV}}$ & $b l a_{\text {CTX-M }}$ & $A m p C_{\mathrm{CIT}}$ \\
\hline Urine & 41 & 19 & 6 & 11 \\
\hline Swab from the wound & 29 & 10 & 3 & 7 \\
\hline Swab from cannula & 3 & 1 & 0 & 3 \\
\hline Swab & 23 & 7 & 6 & 6 \\
\hline Sputum & 5 & 1 & 2 & 2 \\
\hline Swab from the throat & 5 & 2 & 2 & 0 \\
\hline Other smear & 14 & 2 & 5 & 2 \\
\hline
\end{tabular}

The data are in absolute terms

\section{Conclusion}

In the clinical isolates of Escherichia coli-producing ESBL which were collected, a high prevalence of resistance against the majority of tested antimicrobial drugs was detected. The only antibiotics that were effective against all tested isolates were carbapenem and meropenem.

The results showed differences not only between the sexes, but also between different age groups. Rate risk in relation to age and diagnosis, pointing out its upward trend with age, more pronounced in the age group 18-40 years, the proportion of the risk of ESBL-positive E. coli strains is double compared to other age groups $(\mathrm{OR}=2.1895 \% \mathrm{CI})$.

We can say that the resistance of Enterobacteriaceae to $\beta$-lactams antibiotic is conditional to production of Extended-Spectrum $\beta$-Lactamases (ESBLs) and is a significant problem contemporary medicine. The major reason is the possibility of treatment failure penicillins and cephalosporins, including preparations with an extended spectrum of action, in the case of infections caused by bacteria with the production of those enzymes.

This leads not only to other infectious complications and prolong the period of hospitalization, but also represents a threat to life. The treatment of infection caused by resistant bacteria strains increases the direct and indirect costs of treatment several fold.

It is necessary early the detection of the present mechanism of resistance and subsequent deployment of adequate antibiotic therapy.

\section{Acknowledgement}

This research was partially supported by the Agency of the Slovak Ministry of Education for the Structural Funds of the EU, under project ITMS: 26220220162

\section{Author's Contributions}

Viera Lovayová and Lucia Vargová: Participated in all experiments, coordinated the data-analysis and contributed to the writing of the manuscript. Designed the research plan and organized the study.
Mária Nagyová: Participated in all experiments, coordinated the data-analysis and coordinated the mouse work.

Eva Dudriková and Erik Dorko: Designed the research plan and organized the study and contribute to the writing of the manuscript.

Leonard Siegfried: Participated in all experiments, designed the research plan and organized the study, coordinated the data-analysis and contribute to the writing of the manuscript.

\section{Ethics}

This article is original and contains unpublished material. The corresponding author confirms that all of the other authors have read and approved the manuscript and no ethical issues involved.

\section{References}

Apisarnthanarak, A., P. Kiratisin, P. Saifon, R. Kitphati and S. Dejsirilert et al., 2007. Clinical and molecular epidemiology of community-onset, extendedspectrum $\beta$-lactamase-producing Escherichia coli infections in Thailand: A case-case-control study. Am. J. Infect. Control, 35: 606-612. DOI: 10.1016/j.ajic.2007.05.008

Bogaerts, P., H. Rodriguez-Villalobos and C. Laurent, 2009. Emergence of extended-spectrum-AmpCexpressing Escherichia coli isolates in Belgian hospitals. J. Antimicrob. Chemother., 63: 1073-1075. DOI: $10.1093 / \mathrm{jac} / \mathrm{dkp} 046$

Bonnet, R., 2004. Growing group of Extended-Spectrum $\beta$-Lactamases: The CTX-M enzymes. Antimicrob. Agents Chemother., 48: 1-14. DOI: 10.1128/AAC.48.1.1-14.2004

Bradford, P.A., 2001. Extended-Spectrum $\beta$-Lactamases in the 21 st century: Characterization, epidemiology and detection of this important resistance threat. Clin. Microbiol. Rev., 14: 933-951. DOI: $10.1128 / \mathrm{CMR} .14 .4 .933-951.2001$

CLSI, 2011. M02-A11 performance standards for antimicrobial disk susceptibility tests. Clinical and Laboratory Standards Institute, Wayne, PA, USA. 
CLSI, 2009a. Performance standards for antimicrobial susceptibility testing; nineteenth informational supplement M100-S19. Clinical and Laboratory Standards Institute, Wayne, PA, USA.

CLSI, 2009b. Performance standards for antimicrobial disk susceptibility tests. Clinical and Laboratory Standards Institute, Wayne, PA, USA.

CLSI, 2006. Performance standards for antimicrobial susceptibility testing: Sixteenth informational supplement. Clinical Laboratory Standards Institute, Wayne, PA, USA.

Coudron, E.P., E.S. Molland and K.S. Thomson, 2000. Occurrence and detection of AmpC beta-lactamases among Escherichia coli, Klebsiella pneumoniae and Proteus mirabilis isolates at a veterans medical center. J. Clin. Microbiol., 38: 1791-1796. PMID: 10790101

Edwards, S.J., C.E. Emmas and H.E. Campbell, 2005. Systematic review comparing meropenem with imipenem plus cilastatin in the treatment of severe infections. Curr. Med. Res. Opin., 21: 785-794. DOI: $10.1185 / 030079905 X 46223$

Freiwald, A. and S. Sauer, 2009. Phylogenetic classification and identification of bacteria by mass spectrometry. Nat. Protoc., 4: 732-742.

DOI: $10.1038 /$ nprot.2009.37

Ghuysen, J.M., 1991. Serine beta-lactamases and penicillinbinding proteins. Annu. Rev. Microbiol., 45: 37-67. DOI: 10.1146/annurev.mi.45.100191.000345

Howard, C., A. van Daal, G. Kelly, J. Schooneveldt and G. Nimmo et al., 2002. Identification and minisequencing-based discrimination of SHV $\beta$ Lactamases in nosocomial infection-associated Klebsiella pneumoniae in brisbane, Australia. Antimicrob. Agents Chemother., 46: 659-664. DOI: 10.1128/AAC.46.3.659-664.2002

Jacoby, G.A. and A.A. Medeiros, 1991. More extendedspectrum $\beta$-lactamases. Antimicrob. Agents Chemother., 35: 1697-1704.

DOI: 10.1128/AAC.35.9.1697

Laupland, K.B., D.B. Gregson, D.L. Church, T. Ross and J.D. Pitout, 2008. Incidence, risk factors and outcomes of Escherichia coli bloodstream infections in a large Canadian region. Clin. Microbiol. Infect., 14: $1041-1047$. DOI: $10.1111 /$ j.1469-0691.2008.02089.x

Martine'z-Martine'z, L., S. Herna'ndez-Alle's, S. Albertı's, J. Toma's and V. Benedi et al., 1996. In vivo selection of porin-deficient mutants of Klebsiella pneumoniae with increased resistance to cefoxitin and expanded-spectrum-cephalosporins. Antimicrob. Agents Chemother., 40: 342-348.
Moland, E.S., J.A. Black, A. Hossain, N.D. Hanson and K.S. Thomson et al., 2003. Discovery of CTX-MLike Extended-Spectrum $\beta$-Lactamases in Escherichia coli Isolates from Five U.S. States. Antimicrob. Agents Chemother., 47: 2382-2383. DOI: 10.1128/AAC.47.7.2382-2383.2003

Munier, G.K., C.L. Johnson, J.W. Snyder, E.S. Moland and N.D. Hanson et al., 2010. Positive ExtendedSpectrum- $\beta$-Lactamase (ESBL) screening results may be due to AmpC $\beta$-lactamases more often than to ESBLs. J. Clin. Microb., 48: 673-674. DOI: 10.1128/JCM.01544-09

Nordmann, P. and L. Poirel, 2002. Emerging carbapenemases in Gram-negative aerobes. Clin. Microb. Infection., 8: 321-331. DOI: 10.1046/j.1469-0691.2002.00401.x

Oteo, J., C. Navarro, E. Cercenado, A. Delgado-Iribarren and I. Wilhelmi et al., 2006. Spread of Escherichia coli strains with high-level cefotaxime and ceftazidime resistance between the community, long-term care facilities and hospital institutions. J. Clin. Microbiol., 44: 2359-2366.

DOI: $10.1128 /$ JCM.00447-06

Pages, J.M., M. Masi and J. Barbe, 2005. Inhibitors of efflux pumps in Gram-negative bacteria. Trends Molecular Med., 11: 382-389.

DOI: 10.1016/j.molmed.2005.06.006

Paterson, D.L., 2006. Resistance in gram-negative bacteria: Enterobacteriaceae. Am. J. Infect. Control., 34: S64-S73. PMID: 16735147

Plaziniski, J., Y.H. Cen and B.G. Rolfe, 1985. Identification of plasmids by gel electrophoresis. Applied Environ. Microbiol., 48: 1001-1003.

Sambrook, J., E.F. Frish and T. Maniatis, 1982. A laboratory manual. Harbor Laboratory press.

Sidjabat, H.E., D.L. Paterson, J.M. Adams-Haduch, L. Ewan and A.W. Pasculle et al., 2009. Molecular epidemiology of CTX-M-producing Escherichia coli isolates at a tertiary medical center in western Pennsylvania. Antimicrob. Agents Chemot., 53: 4733-4739. PMID: 19687234 